\title{
Chemosensitive augmentation effect by combination treatment of vorinostat and arsenic trioxide in U266 cell line
}

\begin{abstract}
Objective: To investigate the chemosensitivity by HDAC inhibitor vorinostat alone, arsenic trioxide (ATO) alone and vorinostat in combination with ATO on U266 cell.

Methods: CCK-8 assay was used to compare U266 cell proliferation in different groups; Annexin V and PI staining by flow cytometry was used to detect apoptosis rate after treatment; Western blot was used to detect P53, Akt, pAkt, Acetyl-Histone in protein expression levels.
\end{abstract}

Results: vorinostat and ATO significantly inhibited the proliferation of U266 cells in a time-and dose-dependent manner. The U266 cells apoptosis rates were $17.90 \pm 2.60 \%$, $26.45 \pm 4.95 \%, 81.60 \pm 4.20 \%$ respectively after 48 hours of vorinostat $(2 \mathrm{umol} / \mathrm{L})$ treatment, ATO $(16 \mathrm{~mol} / \mathrm{L})$ treatment, and ATO $(16 \mathrm{umol} / \mathrm{L})$ combined with $2 \mathrm{umol} / \mathrm{L}$ vorinostat treatment. Significant changes in cell morphology were observed in AO / EB staining under a fluorescence microscope between drug treatment groups and control. vorinostat -treatment group and ATO-treatment group showed obvious condensation nuclei, nuclear fragmentation, while vorinostat in combination with ATO group showed more significant apoptosis. vorinostat in combination with ATO group showed lower level of P53 expression than vorinostat or ATO alone detected by Western blot. Vorinostat group and combination group showed increased level of Acetyl-Histone protein expression.

Conclusions: vorinostat and ATO both inhibit proliferation and induce apoptosis on U266 cells in a time and dose-dependent manner. Vorinostat in combination with ATO can significantly increase the apoptosis rate of U266 cells in a synergistic manner.

Keywords: histone deacetylase inhibitors, arsenic trioxide, U266 cell, vorinostat

\author{
Volume 6 Issue 2 - 2018
}

Xiaoyan Guan, ${ }^{1,2}$ \# Fang Li, ${ }^{1,3 \#}$ Xiaofan Li, Yuanzhong Chen,' Nainong $\mathrm{Li}^{\prime}$

'Department of Hematology, Fujian Institute of Hematology, China

${ }^{2}$ South Hospital(linshan) of Fujian Provincial Hospital, China ${ }^{3}$ Department of Geriatrics, Fudan University Zhongshan Hospital, China

\#These authors contributed equally to this article

Correspondence: Nainong Li, Department of Hematology, Fujian Medical University Union Hospital, Fuzhou P.R.China; 35000I,Tel 86-59I-83357896/804, Email nainli@fjmu.edu.cn

Received: January 27, 2018 | Published: March 28, 2018

\section{Introduction}

Multiple myeloma (MM) is a disorder of terminally differentiated plasma cells characterized by clonal expansion in the bone marrow (BM). It is the second-most common hematologic malignancy. Many novel immunotherapy strategies, such as adoptive cell therapy and monoclonal antibodies, are currently under investigation in clinical trials, with encouraging outcomes in few of the strategies due to their positive impact on patient survival. ${ }^{1}$ Despite significant advances in therapeutic strategies, like new drugs and tandem auto-HSCT, are capable of enhancing the overall survival of the patients, ${ }^{2} \mathrm{MM}$ still poses a challenge and remains a predominantly incurable disease and demands the need for the development of new treatment regimens for better insights of MM. ${ }^{3}$

Histone acetylation and deacetylation comprise one of the common modifications found in epigenetics. Histone acetylation helps transcription factors bind to DNA templates and activates transcription; however, histone deacetylation inhibits transcription. ${ }^{4-7}$ In 2006 , the second generation of hydroxamic acids histone deacetylase inhibitor (HDACi) vorinostat (suberoylanilide hydroxamic acid) was approved by the FDA for clinical treatment of relapsed or refractory cutaneous T-cell lymphoma. ${ }^{8}$
Arsenic trioxide (ATO) has long been used in biomedicine, especially in traditional Chinese medicine and is still used to treat cancer and other conditions. ${ }^{9}$ Arsenic trioxide (ATO) with or without all-trans-retinoicacid (ATRA) has proved highly effective involving patients with newly diagnosed acute promyelocytic leukemia. ${ }^{10}$ Studies also showed reduced hematologic toxicity as compared with ATRA and chemotherapy. ${ }^{11}$ The efficacy of ATO in the management of other hematological and solid tumor malignancies is also being tested. $^{12}$

To better eradicate the MM clone and overcome the drug resistance, we hypothesized that vorinostat augments chemosensitivity when combined with ATO, which can be widely used as chemoreagent for clinical treatment of hematologic malignancies. Thereby, we investigated the anti-leukemia effect of vorinostat combined with ATO on the U266 cell line.

\section{Material and methods}

\section{Reagent and cell lines}

Vorinostat was kindly provided by Dr. Defu Zeng (City of Hope National Medical Center, US, 91010). DMSO was diluted to a concentration of $10 \mathrm{mmol} / \mathrm{L}$. ATO was purchased from Heilongjiang Harbin Medical University Pharmaceutical Co., Ltd., and diluted to 
a concentration of $2 \mathrm{mmol} / \mathrm{L}$. Both vorinostat and ATO were stored at $-20^{\circ} \mathrm{C}$. Roswell Park Memorial Institute (RPMI) 1640 was purchased from Gibco; fetal bovine serum was purchased from Tianjin Haoyang Company; penicillin / streptomycin was purchased from Hyclone; an apoptosis detection kit (Annexin-V-FITC, PI double staining) was purchased from Roche; Protein Extraction reagents were purchased from Wuhan Boster Biological Co., Ltd.; antibodies against Akt pAkt P53 Acetyl-Histone H3 and Acetyl-Histone H4, and other proteins were purchased from Cell Signaling Technology. The U266 cell line was preserved and cultured in Fujian Institute of Hematology with a standard protocol as previously described. ${ }^{13,14}$

\section{Proliferation assay}

A CCK-8 assay was used to detect cell line proliferation. A positive control, negative control and the drug treated group (vorinostat: 0.25 , $0.5,1,2 \mu \mathrm{mol} / \mathrm{L}$; ATO: $1,2,4,8 \mu \mathrm{mol} / \mathrm{L})$ were detected. The assay was performed in triplicate for each group. Next, $5 \times 104$ cells $/ \mathrm{ml}$ were seeded into a 96-well culture plate to a final volume of $100 \mu$ l. After 24,48 , and $72 \mathrm{~h}$ of treatment, $10 \mu \mathrm{l}$ of CCK-8 was added into each well and incubated for $1-4 \mathrm{~h}$ at $37^{\circ} \mathrm{C}$ in a $5 \% \mathrm{CO} 2$ incubator. The absorbance at $450 \mathrm{~nm}$ was measured using a microplate reader. The inhibition rate was calculated using the following equation: Inhibition rate $=[$ (control group - experimental group $) /$ blank group $] \times 100 \%$. A proliferation curve was plotted based on the drug concentration and the proliferation inhibition rate. Synergetic effect of the combination of vorinostat and ATO was analyzed by Jin's formula. Q was calculated using the following equation: $\mathrm{Q}=\mathrm{E}(\mathrm{ab}) /(\mathrm{Ea}+\mathrm{Eb}-\mathrm{Ea} \times \mathrm{Eb})$ as previously described. ${ }^{6,7}$ Generally, Eab, Ea and $\mathrm{Eb}$ are the average effects (inhibition rate) of the combination treatment, vorinostat only, and ATO only, respectively. Q>1.15 was considered to be a synergistic effect, $0.85>\mathrm{Q}>1.15$ an additive effect, and $\mathrm{Q}<0.85$ an antagonistic effect.

\section{Annexin-V and PI staining}

Apoptosis was detected by Annexin-V and PI staining. Generally, U266 cells $(1 \times 106)$ and the appropriate drugs were co-incubated for $48 \mathrm{~h}$. Cells were harvested after a single wash with PBS and binding buffer $(100 \mu l)$ was added to resuspend the cells. Annexin-V $(2 \mu l)$ and PI $(2 \mu 1)$ were added, and the cells were incubated at room temperature for $10-15 \mathrm{~min}$ in the dark. U266 cell were detected by flow cytometry. According to staining of the cell, living cells were difined Annexin- $\mathrm{V}^{-} /$ $\mathrm{PI}^{-}$, early apoptotic cells were difinedAnnexin- $\mathrm{V}^{+} / \mathrm{PI}^{-}$and late apoptotic cells were difined Annexin- $\mathrm{V}^{+} / \mathrm{PI}^{+}$. This experiment was repeated three times.

\section{AO / EB fluorescence staining}

U266 cells and the drugs were co-incubated as described above. Suspended cells $(95 \mu 1,5 \times 106 / \mathrm{ml})$ were mixed with acridine orange/ ethidium bromide(AO/EB)(5 $\mu$ l). Immediately after mixing, one drop of suspended cells was placed on a clean glass slide and observed by fluorescence microscopy at an excitation wavelength of $490 \mathrm{~nm}$. Cells with green fluorescence in the nucleus and cytoplasm were normal cells; cells with yellow-green fluorescence in the nucleus or cytoplasm were apoptotic cells; cells with red fluorescence in nucleus were necrotic cells.

\section{Western blot}

U266 cells were co-cultured as described above. After the cells were lysed, the supernatant was collected and quantified. Total protein $(40 \mu \mathrm{g})$ was transferred to a PVDF membrane after SDSPAGE electrophoresis. After blocking at room temperature for $2 \mathrm{~h}$, the primary antibodies (against P53, Acetyl-Histone H3, Acetyl-Histone $\mathrm{H} 4$, Akt and pAkt) were added and incubated at $4^{\circ} \mathrm{C}$ overnight. After washing, the secondary antibody (horseradish peroxidaseconjugated anti-mouse $\operatorname{IgG}$ ) was added and incubated for $2 \mathrm{~h}$. The chemiluminescence reaction was performed. $\beta$-actin was used as an internal control.

\section{Statistical analysis}

Statistical analysis was performed with SPSS 11.5. Means were compared by the Dunnett-t test, with $\mathrm{P}<0.05$ considered $\mathrm{t}$.

\section{Results}

\section{Vorinostat in combination with ATO showed an additive effect on the inhibition of proliferation}

Compared with the control group, vorinostat and ATO inhibited proliferation of U266 cells in a time- and dose-dependent manner. After $72 \mathrm{~h}$ incubation, both vorinostat and ATO group showed the highest inhibition of proliferation compared to $24 \mathrm{~h}$ and $48 \mathrm{~h}$. In the vorinostat group, the inhibition of proliferation was increased and reached the plateau with the dose from $0.25,0.5,1,2 \mathrm{umol} / \mathrm{L}$. The dose of $2 \mathrm{umol} / 1$ of vorinostat showed the the highest inhibition of proliferation. In the ATO group, the inhibition of proliferation was increased and reached the plateau with the dose from $1,2,4,8 \mu \mathrm{mol} / \mathrm{L}$. The dose of $8 \mathrm{umol} / 1$ of ATO showed the the highest inhibition of proliferation. After the cells were treated with vorinostat and ATO, cell proliferation was significantly inhibited (Figure 1). vorinostat in combination with ATO showed an additive effect on the inhibition of proliferation (Table 1).

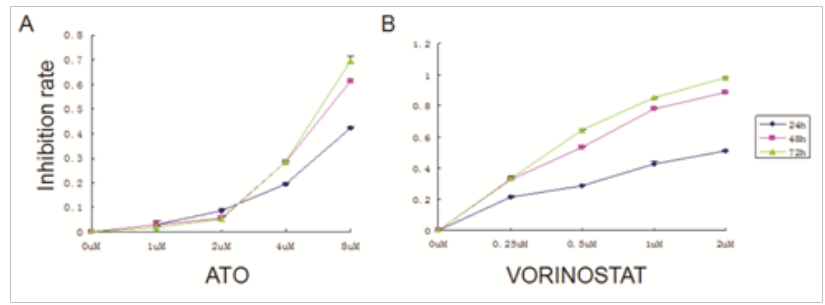

Figure I VORINOSTAT and ATO alone showed inhibition of proliferation on U266 cell line. $5 \times 10^{4}$ cells / $\mathrm{ml}$ were seeded to a 96-well culture plate in a final volume of $100 \mu \mathrm{l}$. After 24,48 , and $72 \mathrm{~h}$ of treatment, I0 $\mathrm{\mu l}$ of CCK-8 was added into each well and incubated for I-4 h. The absorbance at $450 \mathrm{~nm}$ was measured using a microplate reader. Inhibition rate $=[$ (control group experimental group) / blank group] $\times 100 \%$. (A)The proliferation curve of ATO alone is shown. (B)The proliferation curve of VORINOSTAT alone is shown

\section{Vorinostat in combination with ATO showed more apoptosis than the single drugs alone}

Annexin-V-FITC/PI double staining flow cytometry can distinguish early apoptotic cells, late apoptotic cells and necrotic cells. In the lower left quadrant are normal cells; in the lower right quadrant are early apoptotic cells; in the upper right quadrant are late apoptotic cells; in the upper-left quadrant are necrotic cells. The results showed that $95 \%$ of the cells in the control group were living cells; the single drug apoptosis rates increased with increasing drug concentration, suggesting the apoptosis effect is also dose-dependent. The U266 
cells apoptosis rates were $17.90 \pm 2.60 \%, 26.45 \pm 4.95 \%, 81.60 \pm 4.20 \%$, $48 \mathrm{~h}$ after treatment with vorinostat $(2 \mu \mathrm{mol} / \mathrm{L})$, ATO $(16 \mu \mathrm{mol} / \mathrm{L})$ and $16 \mu \mathrm{mol} / \mathrm{L}$ ATO combined with $2 \mu \mathrm{mol} / \mathrm{L}$ vorinostat, respectively. Vorinostat in combination with ATO showed significant apoptosis of U266 cells compared to the single drugs alone $(\mathrm{p}<0.01)$ (Figure $2 \mathrm{~A})$.

The apoptosis results were confirmed by $\mathrm{AO} / \mathrm{EB}$ staining. In the control group, U266 cells showed uniform cell size and morphology and homogeneous green fluorescence in the nucleus and cytoplasm. In contrast, $48 \mathrm{~h}$ after vorinostat and ATO combined treatment, we found differences in cell size and morphology. The nucleus was dense, showing yellow-green fluorescent debris. Additionally, vorinostat in combination with ATO showed more apoptosis characteristics than the single drugs alone (Figure 2B-E).

Table I Vorinostat in combination with ATO showed an additive effect on the inhibition of proliferation

\begin{tabular}{|c|c|c|c|c|}
\hline \multicolumn{2}{|c|}{ Concentration ( $\mu \mathrm{mol} / \mathrm{L})$} & \multirow[t]{2}{*}{ Inhibition (\%) } & \multirow[t]{2}{*}{ Q value } & \multirow[t]{2}{*}{ Additive effect } \\
\hline SAHA & ATO & & & \\
\hline 0 & 0 & 0 & & \\
\hline 0.25 & 0 & 32.73 & & \\
\hline 0.5 & 0 & 53.49 & & \\
\hline I & 0 & 78.04 & & \\
\hline 2 & 0 & 88.56 & & \\
\hline 0 & 1 & 3.17 & & \\
\hline 0 & 2 & 5.48 & & \\
\hline 0 & 4 & 28.33 & & \\
\hline 0 & 8 & 61.38 & & \\
\hline 0.25 & 1 & 37.99 & 1.089 & + \\
\hline 0.25 & 2 & 44.05 & 1.209 & ++ \\
\hline 0.25 & 4 & 55.03 & 1.062 & + \\
\hline 0.5 & I & 60.34 & 1.098 & + \\
\hline 0.5 & 2 & 67.02 & 1.196 & ++ \\
\hline 0.5 & 4 & 65.94 & 0.989 & + \\
\hline I & 1 & 75.91 & 0.964 & + \\
\hline I & 2 & 78.3 & 0.988 & + \\
\hline I & 4 & 83.72 & 0.994 & + \\
\hline
\end{tabular}

A

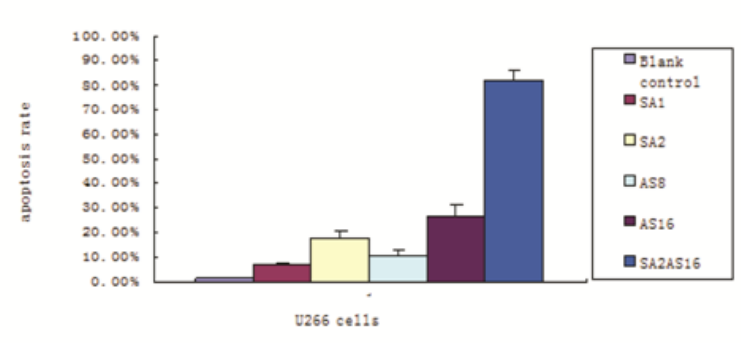

B

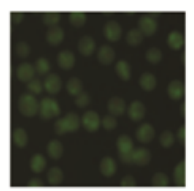

C

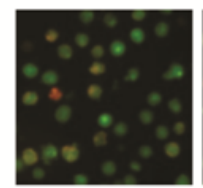

D

E
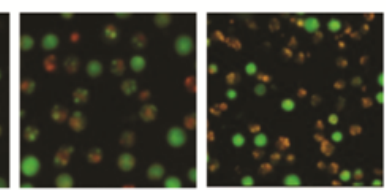

Figure 2 Apoptosis rate after treatment

(A)Apoptosis was detected by Annexin-V and PI staining. U266 cells $(|\times| 06)$ and the drugs were co-incubated for $48 \mathrm{~h}$. Cells were harvested after a single PBS wash. 100 $\mu$ l of binding buffer was added to resuspend the cells. Annexin-V $(2 \mu \mathrm{l})$ and $\mathrm{Pl}(2 \mu \mathrm{l})$ was added. After an incubation at room temperature for $10-15 \mathrm{~min}$ in the dark, U266 cells were detected using a Flow Machine. The apoptosis rates after different treatments are shown (SAI:I $\mu \mathrm{mol} / \mathrm{L} \quad$ VORINOSTAT;SA2:2 $\mu \mathrm{mol} / \mathrm{L} \quad$ VORINOSTAT;AS8: $8 \mu \mathrm{mol} / \mathrm{L}$

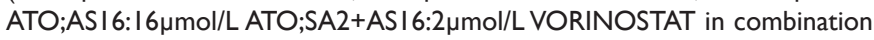
with $16 \mu \mathrm{mol} / \mathrm{LATO}$ ).

(B-E) Apoptosis observations using fluorescence microscopy $48 \mathrm{~h}$ after treatment ( $\times 400$ magnification). U266 cells and the drugs were co-incubated as described in Figure 2. Suspended cells $(95 \mu \mathrm{l}, 5 \times 106 / \mathrm{ml})$ were mixed with

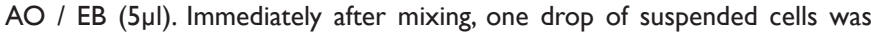
placed on a clean glass slide and observed by fluorescence microscopy at an excitation wavelength of $490 \mathrm{~nm}$. Cells with green fluorescence in the nucleus and cytoplasm were normal cells; Cells with yellow-green fluorescence in the nucleus or cytoplasm were apoptotic cells; cells with red fluorescence in the nucleus were necrotic cells. (B) control;(C) $2 \mu \mathrm{mol} / \mathrm{LVORINOSTAT;}$ (D) $16 \mu \mathrm{mol} /$ L ATO; (E) $2 \mu \mathrm{mol} / \mathrm{L}$ VORINOSTAT in combination with $16 \mu \mathrm{mol} / \mathrm{L}$ ATO.

\section{Vorinostat in combination with ATO showed pronounced changes in protein expression}

Both vorinostat and ATO alone and in the combination group showed lower levels of pAkt expression. The vorinostat group and the combination group showed increased levels of Acetyl-Histone H3 
and Acetyl-Histone $\mathrm{H} 4$ protein expression. There were no significant changes of Akt protein in vorinostat ATO alone and combination group. However, the combination group showed lower levels of pAkt protein expression than vorinostat or ATO alone, suggesting less of the acitivation of Akt when combining ATO and vorinostat together. Interestingly, ATO alone showed higher P53, which can be reversed with the combination of vorinostat (Figure 3).

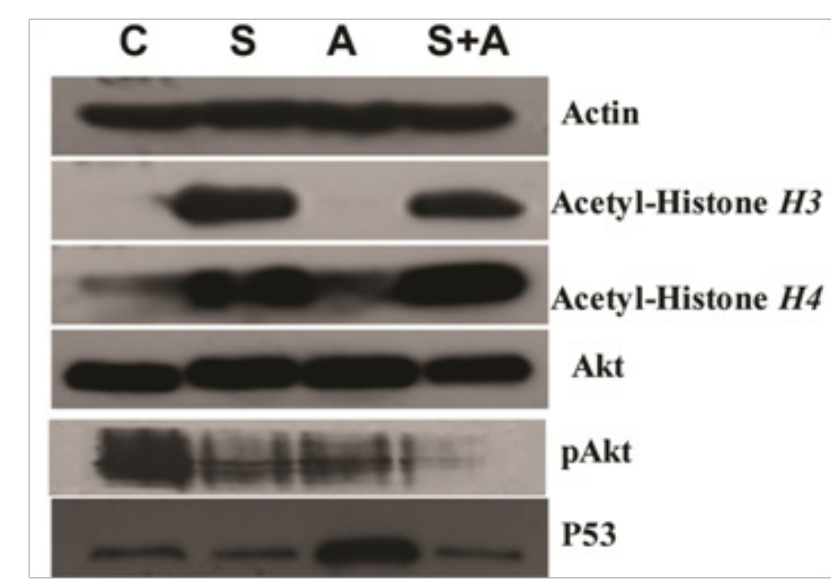

Figure 3 VORINOSTAT in combination with ATO
showed U266 cells were co-cultured as described above. Protein expression was detected by Western blot. $\beta$-actin was used as an internal control. (C. control; S. $2 \mu \mathrm{mol} / L$ VORINOSTAT; A. I $6 \mu \mathrm{mol} / \mathrm{L}$ ATO; S+A. $2 \mu \mathrm{mol} / \mathrm{L}$ VORINOSTAT in combination with $16 \mu \mathrm{mol} / \mathrm{L}$ ATO)

\section{Discussion}

There are a variety of hydroxamic acids histone deacetylase inhibitors (HDACIs) undergoing clinical trials for the treatment of solid tumors and hematological malignancies, especially for the treatment of cutaneous T-cell lymphoma (CTCL), peripheral T-cell lymphoma, and Hodgkin's lymphoma, which have shown promising results. ${ }^{15}$ As a novel HDACI, vorinostat was approved by the FDA for the treatment of progressive clinical recurrence of CTCL.

ATO is an important medicine for treating leukemia. Additionally, chemotherapy regimens based on ATO are topics of research prominence. ${ }^{16}$ In recent years, the mechanism and clinical applications of ATO have been reported, with induction of oxidative stress and DNA damage included. ${ }^{17,18} \mathrm{Lu}$ reported that ATRA and arsenic compound-based combination therapy was effective in reinducing morphological remission in relapsed patients with acute promyelocytic leukemia who had previous exposure to ATRA and arsenic compounds, thus producing low molecular remission rates and high risk of secondary relapse. ${ }^{19}$ Interestingly, we also found chemosensitive augmentation of vorinostat in the NB4 and K562 cell lines ${ }^{20,21}$ which indicates that combined vorinostat and ATO therapy could have clinical applications in the treatment of MM.

The CCK8 results show that individual treatments with vorinostat or ATO inhibited U266 cell proliferation in a time- and dosedependent manner. The combination treatment showed an additive effect in inhibiting proliferation. Additionally, experimental results showed that at certain combined concentrations of vorinostat and ATO, there was a greater level of apoptosis than the single drugs alone, which induced apoptosis over $80 \%$. These results indicated that the combined treatment might have beneficial effects including improved chemotherapeutic effects and minimized side effects.

In this study, both vorinostat and the combination group induced increased expression of Acetyl-Histone H3 and Acetyl-Histone H4 when compared to ATO and the control. This outcome indicates that vorinostat can significantly increase histone acetylation levels and activate transcription, thereby inducing hematological tumor cell apoptosis. ${ }^{6,7}$

Akt is a serine / threonine protein kinase, which plays a major role in the anti-apoptotic pathway in apoptosis signaling. ${ }^{22}$ We found that both the vorinostat group and ATO group down regulate Akt and pAkt expressions. Compared to the single drug treatment, the combination group down regulated Akt and pAkt expressions drastically, indicating that the mechanism may be associated with the regulation of the Akt signaling pathway.

In summary, vorinostat and ATO can significantly inhibit the proliferation of U266 cells and induce apoptosis. Combined treatment can enhance apoptosis effects within a certain concentration range. The mechanism may be associated with increased histone acetylation and the regulation of the Akt signaling pathway. This study may provide a new combination chemotherapy regimen for the treatment of MM. Further in vivo experiments and animal model should be set up to test the enhanced chemosensitivity effect by combining vorinostat and ATO. We also need to observe the side effects by combining vorinostat and ATO and prepare data for translational medicine work.

\section{Acknowledgements}

This work was supported by grants from the National Natural Science Foundation of China (no. 81270641, no.81541024, no. 81200400), Fujian Provincial Grant of Science and Technology (2008F3044, 2016J06018,JA14130, 2016Y9025) and it was Sponsored by National and Fujian Provincial Key Clinical Specialty Discipline Construction Program and Construction project of Fujian medical center of hematology (Min201704).

\section{Conflict of interest}

The author declares no conflict of interest.

\section{References}

1. Dhakal B, Girnius S, Hari P. Recent advances in understanding multiple myeloma. F1000Res. 2016;5.

2. Rajkumar SV. Myeloma today: Disease definitions and treatment advances. Am J Hematol. 2016;91(1):90-100.

3. Rajkumar SV. Multiple myeloma: 2016 update on diagnosis, riskstratification, and management. Am J Hematol. 2016;91(7):719-734.

4. Ciro M Saverio M Pier G P Histone deacetylases and epigenetic therapies of hematological malignancies Pharmacol Res. 2010;62(1):18-34

5. Wang GG, Allis CD, Chi P. Chromatin remodeling and cancer, Part I:Covalent histone modifications. Trends Mol Med. 2007;13(9):363-372.

6. Reid G, Gallais R. Marking time: the dynamic role of chromatin and covalent modification in transcription. Int $J$ Biochem Cell Biol. 2009;41(1):155-163.

7. Victoria MR, Jose G, James SH. Development of vorinostat Current applications and future perspectives for cancer therapy. Cancer Lett. 
2009;280(2):201-210.

8. Siegel D, Hussein M, Belani C, et al. Vorinostat in solid and hematologic malignancies. J Hematol Oncol. 2009;2:31.

9. Zhang XW, Yan XJ, Zhou ZR, et al. Arsenic trioxide controls the fate of the PML-RARalpha oncoprotein by directly binding PML. Science. 2010;328(5975):240-243.

10. Lo-Coco F, Avvisati G, Vignetti M, et al. Retinoic acid and arsenic trioxide for acute promyelocytic leukemia. $N$ Engl J Med. 2013; 369(2):111-121.

11. Nichol JN, Garnier N, Miller WH. Triple A therapy: the molecular underpinnings of the unique sensitivity of leukemic promyelocytes to anthracyclines, all-trans-retinoic acid and arsenic trioxide. Best Pract Res Clin Haematol. 2014;27(1):19-31.

12. Subbarayan PR, Ardalan B. In the war against solid tumors arsenic trioxide needs partners. J Gastrointest Cancer. 2014;45(3):363-371.

13. Liu A, Sun Z, Wang K, et al. Molecular beacon-based fluorescence biosenso for the detection of gene fragment and PCR amplification products related to chronic myelogenous leukemia. Anal Bioanal Chem. 2012;402(2):805812

14. Lin L, Chen J, Lin Q, et al. Electrochemical biosensor based on nanogoldmodified poly-eriochrome black $\mathrm{T}$ film for BCR/ABL fusion gene assay by using hairpin LNA probe. Talanta. 2010;80(5):2113-2119.

15. Duvic M, Talpur R, Ni X, et al. Phase 2 trial of oral vorinostat (suberoylanilide hydroxamic acid, SAHA) for refractory cutaneous T-cell lymphoma (CTCL). Blood. 2007;109(1):31-39.
16. Zhou W, Bao S. PML-mediated signaling and its role in cancer stem cells. Oncogene. 2014;33(12):1475-1484.

17. Sanjay Kumar, Clement G Yedjou, Paul B Tchounwou. Arsenic trioxide induces oxidative stress, DNA damage, and mitochondrial pathway of apoptosis in human leukemia (HL-60) cells. J Exp Clin Cancer Res. 2014;33:42.

18. Wilson H Miller, Hyman M Schipper, Janet S Lee, et al. Mechanisms of Action of Arsenic Trioxide. Cancer Research. 2002;62(14):3893-3903.

19. Lu J, Huang X, Bao L, et al. Treatment outcomes in relapsed acute promyelocytic leukemia patients initially treated with all-transretinoic acid and arsenic compound-based combined therapies. Oncol Lett. 2014;7(1):177-182.

20. Li X, Guan X, Li F, et al. Chemosensitivity enhancement toward arsenic trioxide by inhibition of histone deacetylase in NB4 cell line. J Int Med Res. 2016;44(4):882-892.

21. Li N, Guan X, Li F, et al. Vorinostat enhances chemosensitivity to arsenic trioxide in K562 cell line. PeerJ. 2015;3:e962.

22. Osaki M, Oshimura M, Ito H. PI3K-Akt pathway:its functions and alterations in human cancer. Apoptosis. 2004;9(6):667-676. 